\title{
Simulation Analysis of Stereo Garage Control Algorithm based on ADRC
}

\author{
Yongde Zhang ${ }^{1 *}$, Tongning Shi ${ }^{1}$, Lei Wang ${ }^{1}$, Jingguo $\mathrm{He}^{2}$ and Daifu Tian ${ }^{2}$ \\ ${ }^{1}$ Intelligent Machine Institute, Harbin University of Science and Technology, \\ Harbin, 150080, China \\ ${ }^{2}$ Shangzhi Tiandi Stereoscopic Parking Equipment Manufacturing Co. Ltd., \\ Harbin, 150600, China \\ *Corresponding Author and Email: zhangyd@hrbust.edu.cn
}

\begin{abstract}
With the rapid growth of car ownership, "parking" has gradually become the hot issue of society in general. At present, people widely used stereo garage because it took small area, which had a high space utilization, and convenient operation. However, due to internal instability of three-phase asynchronous motor parameters and load disturbance factors such as fluctuations, it led to a poor robustness of the control system. Therefore, we build a system of first order mathematical model using Simulink based on Active Disturbance Rejection Control (ADRC) and made the simulation analysis by the analysis of the controller structure and algorithm. And through the comparison with the traditional PID control, we implementated the signal fast response optimization design and control overshoot.
\end{abstract}

Keywords: Stereo garage; ADRC; Simulation analysis; PID

\section{Introduction}

With the high pace of developed society, the dependence on traditional PLC control parking equipment gradually exposed the control scope of narrow, poor anti-interference ability. To development and design, therefore, a rich peripherals, strong anti-interference ability, fast processing speed of the control chip applications with three-dimensional garage control, further investigations of the stereo garage controller are more deeply. From the traditional relay control module development to the present intelligent industrial controller, combined with today's network has grown up in the cloud and big data such as high-end technology, makes the relevant technology of the three-dimensional garage controller continued to intelligence, automation, co-ordinated. Three-phase asynchronous motor as a power supply plays an important role in stereo garage, and the run of motor control effect directly determines the work condition of the stereo garage. However, asynchronous motor is a nonlinear, strong coupling system commonly used in the past with the traditional PID control algorithm, which is acknowledged for its easy realization, simple algorithm, and parameter matching, is widely used in industrial control, robot, etc.[1-2] The pattern for errors making in PID controller, however, is not reasonable. The introduction of the integral error feedback is negative, linear combination is not reasonable, and there are many other shortcomings in its application process. Moreover, there is a big limitation, especially in the complex changeable environment, which makes it more difficult to combine PID control and modern control theory to obtain a better control result. Therefore, the essence of this paper is to adopt Active Disturbance Rejection Control (ADRC) to achieve "observation and compensation", at the same time, which will be able to improve the PID error handling commendably, overshoot and

${ }^{*}$ Corresponding Author 
nonlinear problems. We also combined with nonlinear feedback control method to improve the dynamic performance of control system [3-5].

\section{The Overall Design of ADRC}

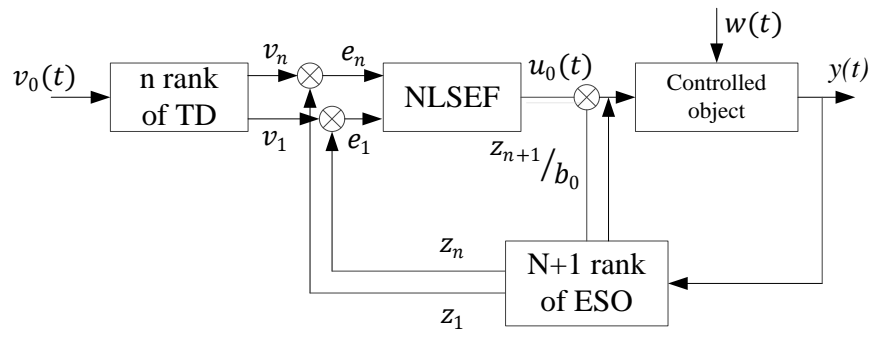

Figure 1. The Overall Design of ADRC

ADRC consists of Nonlinear Tracking Differentiator (NTD), Extended State Observer (ESO) and Nonlinear Error Feedback control (NLSEF), as shown in Figure 1. In this way, we can use the nonlinear transformation to transform the nonlinear system to linear system in an integral tandem structure way, which is in order to realize the dynamic system of feedback linearization. The effect of TD is to extract differential signal and realize the transition process; and ESO is the core part of ADRC, which is to track the observed variables and estimate the uncertain information of system.

\section{The Structure and Algorithm of ADRC}

(1) The Nonlinear Tracking Differentiator (NTD)

The working principle of TD is that if we input $y_{0}(t)$ to TD, we will get to output signals, $y_{1}(t)$ and $y_{2}(t)$. Among them, $y_{1}(t)$ follows $y_{0}(t)$, the differential of $y_{1}(t)$ is $y_{2}(t)$, and regard $y_{2}(t)$ as the approximate differential of $y_{0}(t)$, so in fact, $y_{2}(t)$ is the generalized differential of $y_{0}(t)$.

Discrete control is the most commonly methods in practical control system, we make the foregoing continuous model discretization, so can obtain TD discrete model, the discrete form of tracking differentiator is as shown in the formula (1):

$\left\{\begin{array}{l}y_{1}(k+1)=y_{1}(k)+h \cdot y_{2}(k) \\ y_{2}(k+1)=y_{2}(k)+h \cdot f S t\left[y_{1}(k)-y_{0}(k), x_{2}(k), r, h\right]\end{array}\right.$

(1)

Among them, $h$ represents for the integral step, $y_{0}(t)$ represents for input signal, $f s t()$ represents for nonlinear function, we defined as follows:

$$
d=r \cdot h
$$

$y_{1}(t)$ follows $y_{0}(t), y_{2}(t)$ follows the derivative of $y_{0}(t), r$ and $h$ are adjustable parameters, the follow speed will turn up when we add the value of $r$, and the effect of filtering will be better if we add the value of $h$. But the value of both parameters are conflicting, so it is necessary to coordinate these parameters.

We get a new variable $h_{0}$ as a separate integral step from $h$ in $f s t()$, that we can get fast discrete tracking differentiator as shown in the formula (2): 
$\left\{\begin{array}{l}y_{1}(k+1)=y_{1}(k)+h \cdot y_{2}(k) \\ y_{2}(k+1)=y_{2}(k)+h \cdot f S t\left[y_{1}(k)-y_{0}(k), x_{2}(k), r, h_{0}\right]\end{array}\right.$

(2)

\section{(2) Extended State Observer (ESO)}

As a core component of ADRC and ESO its purpose is to realize the measurement of state variables and its law order differential and the reasonable track, at the same time also need to accurately forecast system uncertain parameters. Observed TD integral method are used to get the quantity of the differential, take this way to effectively solve the observed due to the amount of noise, the differential method is not usually can the problem of noise reduction. The offline form of ESO can be expressed as shown in the formula (3):

$$
\left\{\begin{array}{l}
e_{1}(k)=z_{1}(k)-y(k) \\
z_{1}(k+1)=z_{2}(k)+h\left[z_{2}(k)-\beta_{01} e_{1}\right] \\
z_{2}(k+1)=z_{3}(k)+h\left[z_{3}(k)-\beta_{02} f a l\left(e_{1}, a_{1}, \delta\right)+b_{0} u\right] \\
z_{3}(\kappa+1)=z_{3}(k)-h \beta_{03} f a l\left(e_{1}, a_{2}, \delta\right)
\end{array}\right.
$$

From the narrative above can be concluded that the total disturbance in ESO system including the system's external disturbance and the disturbance within the system itself. Through the nonlinear combination of system state and extended state observer "total disturbance" instant forecast analysis, as well as the appropriate compensation "disturbance", the unknown disturbance of the nonlinear, uncertain object is transformed into the integral series linear control object, the realization of control system for dynamic feedback linearization, the nonlinear, uncertain system gradually to linearization and determine linearization, later will be able to use existing classical control theory to control.

\section{(3) Nonlinear Error Feedback control (NLSEF)}

To sum up, in the application of TD to optimize PID and use the ESO implementation "state feedback", "model and the outer circle compensation" under the basis of all the information you need to get NLSEF. In the structure of NLSEF, because the extended state observer and system model of the unknown disturbances can be instantly make proper compensations to function. NLSEF with non-smooth feedback method, the steadystate error rendering index form into an order of magnitude lower, so only need by using proportional and differential link to improve the controller, to prevent the side effects of integral effect. The extended state observer and tracking differentiator between the output errors can be expressed as:

$$
e_{1}=x_{1}-z_{1}, e_{2}=x_{2}-z_{2}, \ldots \ldots, e_{n}=x_{n}-Z_{n}
$$

Use these error "nonlinear configuration" to realize the nonlinear state error feedback, as shown in the formula (4):

$u_{0}=\beta_{1} \cdot \operatorname{fal}\left(e_{1}, a, \delta\right) \ldots+\beta_{n} \cdot f a l\left(e_{n}, a, \delta\right)$

(4)

Among them, 


$$
f a l(e, a, \delta)=\left\{\begin{array}{l}
|e|^{a} \operatorname{sgn}(e),|e|>\delta \\
e / \delta^{1-a},|e| \leq \delta
\end{array}\right.
$$

And $\beta_{1} 、 \beta_{2} 、 \ldots \beta_{n}$ is adjustable parameters. Formula (4) determines the control of the nonlinear controller, here refers to the controller is independent of the object model, so it is called nonlinear state error feedback control law. If it exists $a<1$, then the function $f a l()$ has a characteristic which is little errors with a big gain or big errors with a little gain. So what we can do is to choose parameter $\beta_{1}, \beta_{2}, \ldots \beta_{n} 、 \delta 、 a$ to obtain rapid adjustment effect.

\section{The Application of the ADRC in Stereo Garage}

Considering the influence of the parameters of three asynchronous motor stator and rotor to decoupling failure caused by conditions of perturbation [6], at the same time within the PID adjustment, etc are not able to fully consider, so we can't put all kinds of uncertainty of the controlled objects condition for effective estimation and compensation, finally makes the three-phase asynchronous motor control performance is affected by the obvious. To improve the control performance of three-phase asynchronous motor speed regulation system, this article is based on the basic principle of ADRC control system for three-phase asynchronous motor control scheme is presented [7-8]. The mathematical model of three-phase asynchronous motor can be represented as shown in Figure 2.

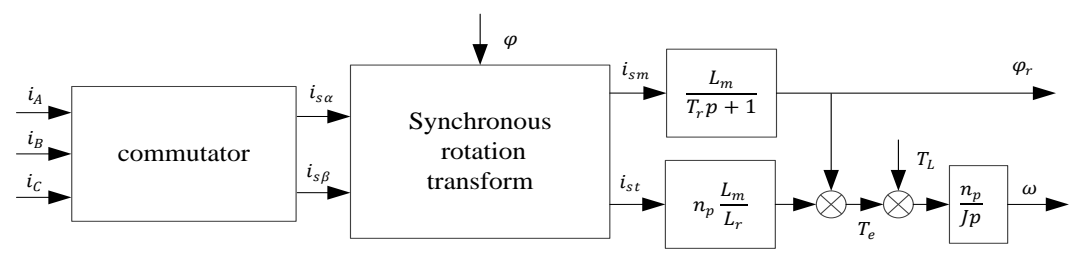

\section{Figure 2. Mathematical Model of Three-Phase Asynchronous Motor}

Control for three phase asynchronous motor drive system, using the first order and second order ADRC controller structure of ESO. In the vector control of motor, usually is to guarantee the rotor flux constant, combined with the characteristics of the ADRC, for the moment of inertia system disturbance and load disturbance caused by such impact due to expansion $2 \mathrm{z}$ tracking compensation, this constitutes the first-order ADRC implementation of three-phase asynchronous motor speed control system, motor speed control based on first-order ADRC principle block diagram is shown in Figure 3.

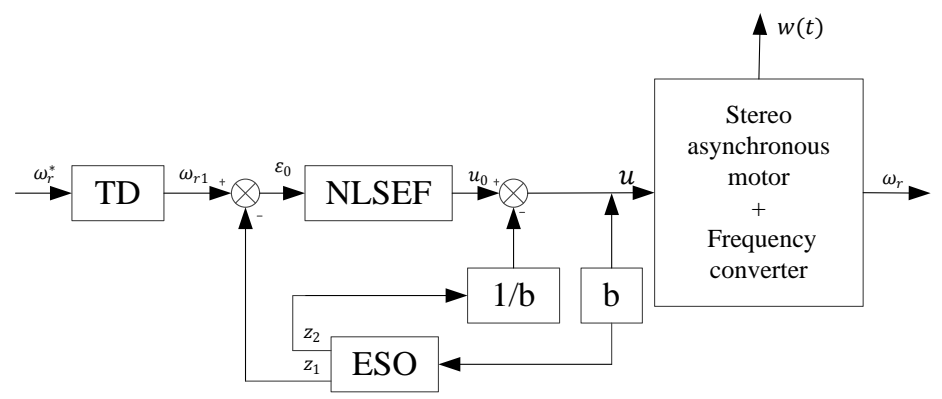

\section{Figure 3. Schematic Diagram of Motor Speed Control based on the First-} Order ADRC 


\section{The Simulation Analysis}

In order to fully verify the control performance of ADRC, we use the Simulink module in MATLAB to make the analysis [9-10]. In order to better illustrate its superiority, we use the PID regulation to analysis the same control object. By comparing the control results, we can better display the control effect. Assume that the non-linear mode of the system is as shown in the formula (5):

$$
f(t)=\operatorname{sign}(\sin (t))
$$

Next, we set the controller parameters as below:

\section{(1) Nonlinear Tracking Differentiator (NTD)}

Here, $\mathrm{r}$ represents for tracking speed factor; h represents for filter factor; $\delta$ represents for linear interval size, we usually selected as the $1 / 100$ of the input signal variation range. Set as

$$
r=10, h=0.01, \delta=0.01
$$

\section{(2) Extended State Observer (ESO)}

$\beta_{01} 、 \beta_{02}$ and $\beta_{03}$ are state error feedback gain parameters, the relationship of them is: $\beta_{01}>0, \beta_{02}>0, \beta_{03}>0$ and $\beta_{01} \cdot \beta_{02}>\beta_{03}, \beta_{01} 、 \beta_{02}$ are mainly affects the observed velocity of the ESO, and the 01 size also depends on the system of sampling time. The $\beta_{01}$ larger, the tracking input signal speed of $z_{2}$ faster. But $\beta_{01} 、 \beta_{02}$ should not to be so large, when they are to large the input signal will include noise ,and it will influence the observation effect of ESO. The $\beta_{03}$ mainly affects the accuracy of the system, when the value of $\beta_{03}$ grows up, even though it will accelerate the estimation speed of the disturbance, it may oscillate and reduced accuracy; when the value of $\beta_{03}$ turns down, it will improve the accuracy of the system, but it will slow down the estimation speed of the disturbance. Set as:

$$
\beta_{01}=100, \beta_{02}=65, \beta_{03}=85, b=1 \text {. }
$$

(3) Nonlinear state error feedback control rate (NLSEF)

Parameter $\beta_{1} 、 \beta_{2}$ corresponds to the parameter $\mathrm{P}, \mathrm{D}$ of the PID regulation, which is the same characteristics. The value of $\alpha$ generally taken to be 0.5 . Set as:

$$
\beta_{1}=100, \beta_{2}=10, a=0.5
$$

In this paper, we use the S-Function function to write TD, ESO and various function modules of the ADRC, and build Simulink simulation diagram, as shown in Figure 4. In order to illustrate the advantage of ADRC in the fast response and overshoot control better, the paper also designed a PID controller emulation, as shown in Figure 5: 


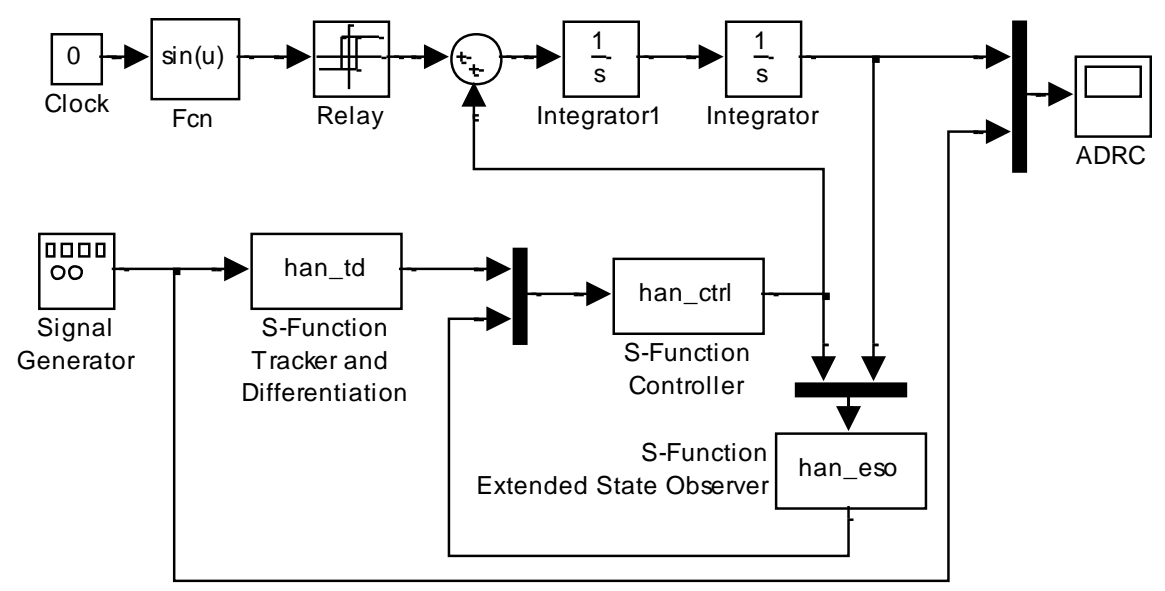

Figure 4. Block Diagram of ADRC Simulation in Simulink

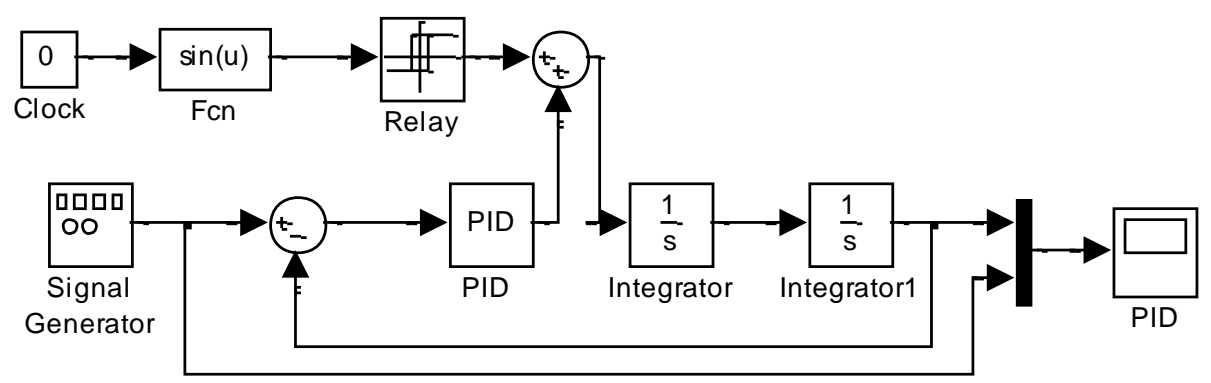

Figure 5. Block Diagram of PID Simulation in Simulink

This paper adopt ADRC adjustment mode and PID adjustment mode from sinusoidal input signal (Figure 6), the square wave signal input (Figure 7), the saw-tooth signal input (Figure 8) and other aspects to simulate the control object in Simulink.

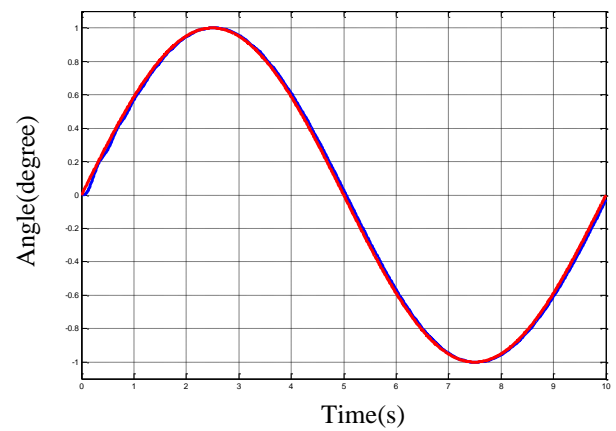

(a) ADRC

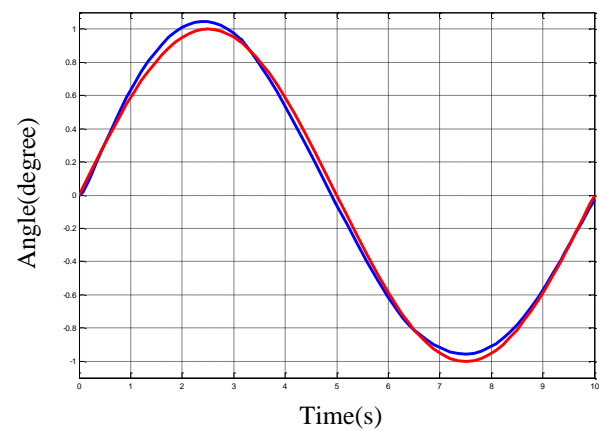

(b) PID

Figure 6. Sinusoidal Response Curve 


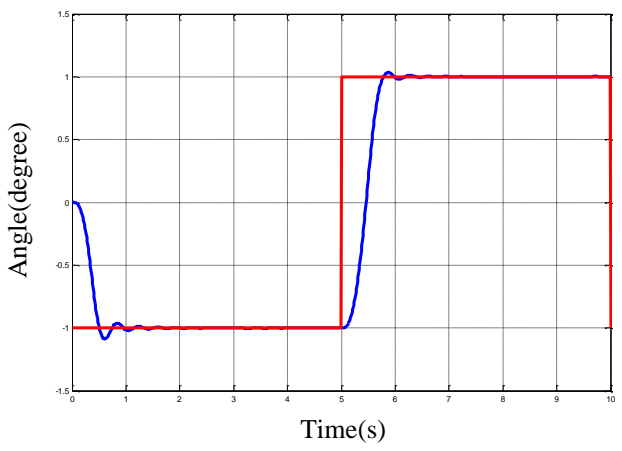

(a) ADRC

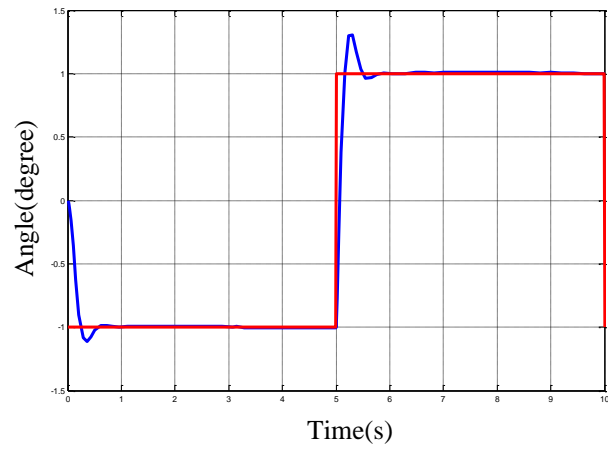

(b) PID

Figure 7. Square Response Curve

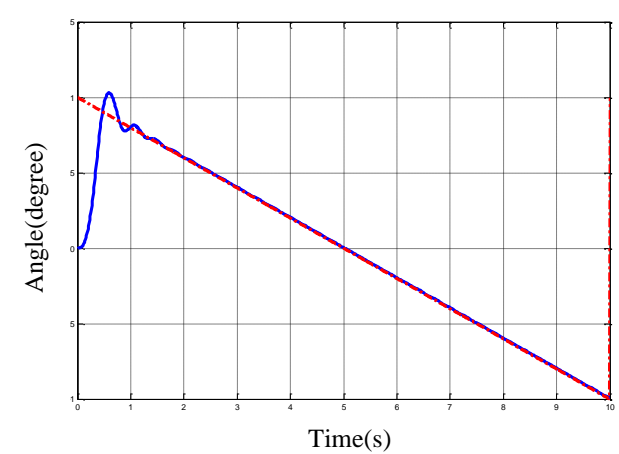

(a) ADRC

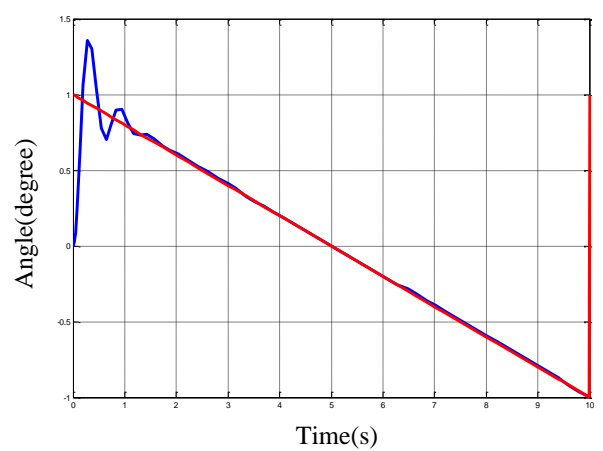

(b) PID

Figure 8. Saw-Tooth Response Curve

In the figure, Figure (a) refers to the ADRC response curves, Figure (b) refers to the PID response curves, where the red line on behalf of a given curve, the blue line represents the response curve. Contrast can be seen from the above Figures the ADRC does not occur overshoot when rapid response, so that a good solution to the conflict between rapid response and overshoot, and can meet the needs of the control system. Although the PID regulator can also achieve the purpose of rapid response, when the rapid response happened the overshoot get larger. The results of ADRC is superior to PID regulation in the error processing, overshoot and nonlinear problems, simulation experiments show that ADRC has good robustness and stability, and ultimately be able to meet the requirements of the actual control system.

\section{Conclusion}

In this paper, by analyzing the fundamental principle and structure of ADRC, the basic composition and the related algorithm of ADRC has been investigated. By comparing with classical PID regulation, it is can be concluded that ADRC is a better way to study Electric machinery in stereo garage. Then asynchronous motor mathematical model was described and motor speed control scheme was designed based on the first order ADRC combination with mathematical control of ADRC. In order to better illustrate ADRC strength in quick response and overshoot control and so on, Simulink module in MATLAB was used to make the simulation analysis of control performance. And then simulation results obtained from the comparison between ADRC regulation and PID regulation verified our theoretical analysis. 


\section{Acknowledgements}

This paper is totally supported by Science and Technology Department of Heilongjiang Province (GC13A410).

\section{References}

[1] Q. Zhang, "The design and application of automated Stereo Garage control system [D]", Chengdu: University of Electronic Science and Technology of China, (2009), pp. 17-21 (in Chinese).

[2] Z. Liu, "Research on Motor Control System of Parking Deck [D]", Tianjin, Tianjin University of Technology, (2008) (in Chinese).

[3] Sayem, A. H. M., "Performance enhancement of ADRC using RC for load frequency control of power system[J]", Proceedings of the 2013 IEEE 8th Conference on Industrial Electronics and Applications, ICIEA 2013, (2013), pp. 433-438.

[4] Fadali, M.S., "On the stability of Han's ADRC [J]", Proceedings of the American Control Conference, (2014), pp. 3597-3601.

[5] J. Hu, "Study of three-motor Synchronous Control System Based on Active Disturbances Rejection Control [D]", Jiangsu, Jiangsu University, (2010) (in Chinese).

[6] W. Wu, X. Huang and H. Liu, "Three-phase AC Induction Motor Control System Simulation [J]", Computing Technology and Automation, no. 4, 2011 (in Chinese).

[7] M. Zhang, "Simulation and Application of Intelligent PID Control [D]", Wuhan Huazhong University of Science and Technology, (2005) (in Chinese).

[8] S. Wang, Y. Shuai and Z. Feng, "A Method for Controlling a Loading System Based on a Fuzzy PID Controller [J]", Mechanical Science and Technology for Aerospace Engineering, vol. 1, (2011), pp. 166172 (in Chinese).

[9] C. Liu and J. Liu, "Analysis of Stepper Motor Close-Loop Control System Based on Simulink, [J]", Measurement and Control Technology, no. 1, (2009), (in Chinese).

[10] A. W. M. (Jos) van Schijndel, "A review of the application of Simulink S-functions to multi domain modelling and building simulation [J]", Journal of Building Performance Simulation, (2014), 73. 